\title{
EL ARGUMENTO DE LA SIMPLICIDAD Y LOS CONCEPTOS DE ESPACIO Y TIEMPO EN SAMUEL CLARKE
}

\section{Laura Benítez Grobert}

\section{Introducción}

A pesar de las controversias sobre el verdadero papel de las propuestas de Clarke en defensa del newtonianismo, es indudable que, por diversas razones, la atención de los especialistas no ha podido soslayar a este autor que, para algunos, resulta sobrevalorado en su propio tiempo ${ }^{1}$, en tanto que, para otros: “[...] llegó muy tarde a la filosofía sistemática y no poseyó el genio de las grandes figuras"2, todo lo cual no obsta para considerar que su sistema es significativo, en vista de que combina aspectos de ciencia y teología, de tal manera que ilumina la génesis de la ciencia nueva. Así, en mi opinión, la filosofía de Clarke es importante no sólo en función de su decidido apoyo y difusión de las ideas de Newton, sino porque su compromiso cientifico, con una propuesta de vanguardia en torno a la materia, se vincula a tres interesantes y no necesariamente ortodoxas propuestas metafísicas, a saber:

1. La simplicidad del espacio y del tiempo

2. Un matizado y peculiar dualismo materia-espíritu

3. Una interesante concepción de la libertad del espíritu

Para articular estas tres ideas, que considero centrales en el pensamiento de Clarke, tomaré como eje el argumento de la simplicidad,

\footnotetext{
* Investigadora titular B del Instituto de Investigaciones Filosóficas de la UNAM. grobet@servidor.unam.mx

1.John H Gay señala en su "Matter and Freedom in the Thought of Samuel Clarke" que, según Coleridge, "toda generación cuenta con uno o más hombres sobrevalorados y Clarke lo fue en el reinado de Jorge I" Joumal of the History of Ideas, Vol. 24. Temple University, Philadelphia, 1963, p. 85.

${ }^{2}$ Gay, John H., op. cit., p. 105.
} 
siguiendo la sugerencia de Mijuskovicz ${ }^{3}$ para quien el argumento de la simplicidad mantiene una integración estable en el pensamiento de occidente y es fundamental para comprender el desarrollo de las ideas en los siglos XVII y XVIII, pues impregna su atmósfera intelectual.

El argumento de la simplicidad nació, básicamente, en relación con la prueba de la inmortalidad del alma. Lo inmaterial es inextenso, luego es simple, luego es indestructible. Siguiendo un uso que podemos llamar 'ortodoxo' del argumento, Descartes, entre otros autores, consideró que el alma es inextensa e inmaterial y tiene, como característica esencial, el pensamiento. Frente a esta presentación, Henry More, el neoplatónico de Cambridge, propone una variación curiosa del argumento. En efecto, en su Inmortalidad del alma, publicada en 1659, define en el axioma 9, como propiedades inmediatas del espíritu o sustancia inmaterial, la penetrabilidad y la indisolubilidad. Su idea general es que todo lo existente es extenso y ello abarca sustancias corpóreas y sustancias pensantes, que se diferencian porque las primeras son divisibles y las segundas no. En suma, disocia inmaterial de inextenso; lo simple es indiviso pero extenso. El significado de simple se restringe a indisoluble, con lo que cabrían formas indivisibles de extensión, precisamente las espirituales.

En el lado racionalista, Leibniz se suma a la idea del alma como unidad indivisible, como una sustancia simple, sin partes, sin extensión, ni figura, ni divisibilidad.

Frente al uso que los racionalistas hicieron del argumento de la simplicidad, surge una propuesta cercana a la de More. Samuel Clarke (16751729), en respuesta a Dodwell, quien afirmaba que el alma es naturalmente mortal, en 1714 sostuvo que el alma es una sustancia creada inmortal, que no es compuesta ni corruptible, esto es, básicamente simple; sin embargo, siguiendo a More afirma que:

${ }^{3}$ Mijuskovicz, Ben Lazare, The Acbilles of Rationalists Arguments, Martinus Nijhoff, The Hague, 1974. 
[...] si las operaciones o acciones de una sustancia no son sino sus modificaciones, los espíritus deben estar sustancialmente presentes donde operan y, en consecuencia, deben ser extensos ${ }^{4}$.

Así, resulta que el alma es simple y a la vez extensa. Pero, ¿qué es lo que hace que la extensión se incluya como parte definitoria de entes espirituales? Al paso que la tradición cartesiana entendía que la extensión está ligada a la materia, para More y para Clarke, la extensión está ligada a la existencia, por lo que el concepto extensión adquiere una mayor amplitud, al abarcar lo corpóreo y lo no corpóreo. Ahora bien, el hecho notable es que, al paso que la extensión corpórea es divisible, la incorpórea es indivisible. Hay pues, ontológicamente, para estos autores, dos órdenes de extensión pero, ¿para qué? ¿En qué dirección apunta semejante distinción? ¿Por qué no conformarse con la división material-espiritual descrita por el par compuesto-simple de vieja prosapia platónica? ¿Por qué añadir la nota de extenso a lo simple, creando con ello la perplejidad de monistas y dualistas?

Me parece que la motivación de Clarke es doble. En efecto, hay en él un motivo teológico, el de la libertad de Dios y del alma, que le lleva a buscar una tesis sobre el mundo natural que no cancele su preocupación por la acción no sujeta a la necesidad ${ }^{5}$. Sin embargo, considero que, a esta preocupación, se suma un problema que surge en el campo de la metafísica y que es el de la noción de acción a distancia, en el sentido de que, como le dice a Leibniz: "[...] nada puede actuar o recibir la acción donde no está"6.

La otra motivación es de carácter científico. Clarke adopta una noción de espacio físico, como extenso pero indivisible, que le sirve tanto en el campo de la filosofía natural como para reforzar sus ideas acerca de las entidades espirituales.

4 Vailati, Ezio, "Clarke's Extended Soul", Joumal for the History of Philosophy, 31 (3), julio, 1993 , p. 387.

${ }^{5}$ Esta propuesta la hace John Gay en su "Matter and Freedom".

6 Vailati cita a Grant quien explica en Much Ado About Notbing que: “... el principio de que la acción requiere contacto espacial entre el agente y el paciente fue aceptado por Tomás de Aquino y por Francisco Suárez como metafísicamente necesario y consecuentemente aplicable a Dios y a sus criaturas; por contraste, Duns Escoto, negó que se aplicara a Dios cuya voluntad es suficiente para conseguir el efecto deseado (Vailati, op. cit., nota 7, p. 389). 
En suma, tenemos que, para Clarke, hay una extensión corpórea material, compuesta, corruptible, degradable, etc., y una extensión incorpórea. Ella comprende entidades o sustancias como los entes espirituales, almas y otros entes creados pero, también, estará el espacio, que no se confunde con los entes espirituales, porque no es estrictamente hablando una sustancia sino, más bien, el efecto de una sustancia eterna.

Lo interesante en este recuento ontológico, es que no sólo se da cabida a un nuevo tipo de entidad, lo cual comenzó desde el Renacimiento buscando la ubicación del espacio como independiente de la materia ${ }^{7}$, sino que se introduce un nuevo matiz, ya que no se trata de nada creado, pues no es ni sustancia ni accidente, sino de una especie de efecto, caracterización que acarrea dificultades para su ubicación y denominación pero que, a la par, abre nuevas posibilidades de explicación que se anclan en el argumento de la simplicidad. Así, al paso que el alma es un simple extenso, pero indivisible, la extensión es una curiosa entidad que se reconoce como simple y de suyo indivisible. A primera vista, tal parece que lo anímico adquiere una nota tradicionalmente material (la de extensión), en tanto que el universo adquiere una nota espiritual (la de simplicidad) para el espacio, que se suma a los entes materiales compuestos que lo conforman.

\section{El argumento de la simplicidad y el dualismo de Samuel Clarke}

En su Discurso sobre el ser y los atributos de Dios, Clarke afirma que

La idea de Dios es una idea primaria y simple [...] En nuestra mente está la idea de un ser máximamente simple, eterno e infinito, original e independiente ${ }^{8}$.

Ésta es justamente la idea de "ser existente de suyo", independiente en cuanto no tiene causa externa, sino que tiene su razón o fundamento en la necesidad de su propia naturaleza. Pero recuérdese que no se trata tan

\footnotetext{
${ }^{7}$ Recordar las propuestas de Francesco Patrizi y Giordano Bruno al respecto.

${ }^{8}$ Clarke, Samuel, "A Discourse Concerning The Being and Attributes of God", en Samuel Clarke. The Works. 1738, Garland Publishing Inc., New York-London, 1978, Vol II, Prop. III, p. 528.
} 
sólo de describir los atributos de la divinidad sino de hacer patente su actividad en el universo. Así, dice a Leibniz:

Siendo Dios omnipotente, está realmente presente a todas las cosas, esencialmente y sustancialmente. Su presencia se manifiesta, ella misma, por su operación, pero no puede operar si no está allí.

Como lo señala Joel M. Rodney, en su artículo sobre Clarke,

Los sermones de Clarke de 1705 estaban dirigidos a los deístas; su reivindicación de la religión natural demostraba, no sólo la existencia de Dios, sino su presencia en el mundo ${ }^{10}$.

Así, luchar contra deístas, ateos, materialistas, etc., fue, en buena parte, su cruzada personal pero, justamente, el carácter específico de la misma estriba en que no se conforma meramente con demostrar la existencia del ser supremo, sino que intenta dar algo más; desea poner en claro que esta presencia de Dios en el mundo no es ociosa o indiferente pues:

El existente de suyo y causa original de todas las cosas, no es un agente necesario, sino un ser dotado de libertad y elección ... [ya que sin ello no habrá] causa, motor, principio o principio del movimiento en ninguna parte ${ }^{11}$.

Es verdad que, según lo dicho por Grant, el principio de acción requiere contacto espacial entre el agente y el paciente, pero ello no implica que Dios requiera de extensión para cumplir con este dictum metafísico. Con todo, Clarke no fue tan obvio; en efecto, en su Cuarta Respuesta a Leibniz, afirma que sólo el vulgo puede creer que Dios necesita un lugar como una entidad previa a Él. No obstante, la propuesta de que Dios necesita estar realmente presente a todas las cosas como condición de su acción, plantea algunas dificultades.

9 The Leibniz-Clarke Correspondence, Ed. H.G. Alexander, Manchester University Press, Barnes \& Noble, New York, 1956 (C-L III,12, pp. 33-34).

10 Rodney, Joel M., "Clarke, Samuel", en Dictionary of Scientific Biograpby, Vol. 3, Charles Scribner's Sons, Nueva York, 1981, p. 295.

${ }^{11}$ Rodney, Joel, op. cit., p. 295. 
La pregunta es, ¿por qué Dios no puede, según Clarke, estar presente sólo por operación y esencia en el universo, como podrían sostenerlo Descartes o Leibniz?

La raíz del problema parece ser, evitar la acción a distancia, incluso para Dios, que no puede actuar donde no está. Pero, como tampoco requiere trasladarse, la cuestión quedaría resuelta recurriendo a su inmensidad, que le hace presente ipso-facto a todas partes. Para Clarke, la inmensidad de Dios, como un peculiar atributo, le permite estar realmente presente a todo el universo:

Para Clarke, el espacio [...] es una propiedad o modo de ser de Dios, mediante la que Él está presente a su creación ${ }^{12}$.

Ahora bien, como la presencia se traduce inmediatamente en acción ¿por qué se necesita la acción inmediata y continua de Dios en el universo? Leibniz lamentaba que el Dios de los newtonianos no fuera providente, en el sentido de que, una vez creada con toda previsión, la máquina del universo no requiriera un constante reajuste por parte de la divinidad. La cuestión es cómo se concibe el universo y, más particularmente, la materia que, para Clarke:

Tiene propiedades pero no poderes; extensión y masa, pero no vitalidad; existe en el espacio vacío y, no obstante, el espíritu está inmediatamente presente a ella a través del medio del espacio. Es capaz de moverse, pero sólo bajo la influencia del espíritu, porque incluso la gravitación universal es, en última instancia, no una propiedad de la materia sino, más bien, el resultado de la acción libre de Dios ${ }^{13}$.

Tal como Gay nos los muestra, la materia es totalmente inerte per se y requiere de la acción divina para iniciar y no decaer en su actividad o movimiento ${ }^{14}$.

12 Gay, John H., op. cit., p. 96.

${ }^{13}$ Ibid., p. 99.

${ }^{14}$ Cfr. Ibid., p. 98, donde Gay cita la parte II, 223 del Discourse Concerming the Being and the Attributes of God: "La materia torpe y carente de vida es, finalmente, incapaz de obedecer ningunas leyes o ser dotada de algún poder y [...] no obstante, el orden y disposición de las cosas que vulgarmente llaman el curso de la naturaleza, no es 
En suma, la causa o principio del movimiento del universo es Dios y no un eslabón más de la cadena causal material, sino un ser espiritual que, como 'causa libre', es la auténtica causa eficiente, en el sentido de dotar de poder a una cadena de eventos materiales, como lo explica Gay.

Así, existe un ser de máxima simplicidad que actúa a través de su atributo espacial infinito, por el que está presente a todas las cosas del universo, el cual dota, por su libre voluntad, de poder a la materia contingente y actúa como una causa no mecánica sobre el universo y desata la acción de las causas materiales que obedecen las leyes del movimiento.

Como puede observarse, Clarke no está en mejor situación que Descartes, cuando explicaba a Elisabeth de Bohemia que las causas mecánicas sólo son aplicables a los cuerpos, pero que la acción entre Dios y el mundo o el alma y el cuerpo debe entenderse como la acción de causas no mecánicas sobre la materia. El gran problema fue, con Descartes, y seguía siéndolo en la época de Clarke, la dificultad para demostrar estas causas, lo cual refleja finalmente, que ninguno de los dos autores pudo realmente solucionar los problemas de interacción que planteaban sus dualismos, en el fondo radicales, aunque con diversos matices. Ello no quita sin embargo que, en el caso de Clarke, se iluminen, a partir del argumento de la simplicidad, la inmensidad de Dios como extensión indivisa y la simplicidad del espacio, que fue un principio metafísico del newtonianismo.

\section{Las nociones físicas de espacio y tiempo y el argumento de la simplicidad}

Dice que el espacio es el orden de las coexistencias y el tiempo el orden de las existencias sucesivas. Supongo que quiere decir que el espacio es el orden de las coexistencias en el espacio y el tiempo el orden de las existencias sucesivas en el tiempo; o que el espacio es espacio en el espacio y el tiempo, tiempo en el tiempo. (Newton a Conti 1716) ${ }^{15}$

posible que sea ninguna otra cosa sino la arbitraria voluntad y el poder de Dios continuamente ejercido y actuado sobre la materia.

${ }^{15}$ Koyré, Alexandre y Cohen, Bernard: 'Newton et la Correspondence LeibnizClarke" en Archive International d'Histoire des Sciences, 15, 1962, p. 71. Cabe mencionar que esta versión no fue la que finalmente envió Newton a Conti, sino que se trata de uno de los tantos borradores encontrados entre sus papeles. 
En la proposición V del Discurso concerniente al Ser y los atributos de Dios, Clarke afirma que, aunque la esencia del ser existente de suyo se nos escapa, sí podemos conocer su existencia y, con ella, varios de sus atributos que son demostrables. Entre éstos, el más importante es que se trata de un ser eterno. El problema epistemológico es grande, no podemos comprender cabalmente la eternidad, pero sí la podemos concebir y demostrar. En realidad basta, en la perspectiva teológica del autor, con aceptar la existencia de Dios, pues ella implica su eternidad pero, además, para Clarke, se puede demostrar. En efecto, como la idea de Dios es una idea primaria y simple, está en nuestra mente como eterno e infinito:

Que la única idea verdadera de un ser existente de suyo o necesariamente existente, es la idea de un ser del que la suposición de su no existencia es una contradicción expresa. Quien supone que no hay un ser eterno e infinito en el universo, supone una contradicción ${ }^{16}$.

La idea es que tales atributos se derivan de su existencia autocreadora e independiente. El atributo de infinitud o inmensidad es sólo predicable de Dios, pero no de la materia. Así, atribuir extensión abstracta y esencial a la materia es hacer de la materia algo infinito, como le ocurre a Descartes, siendo que la materia es contingente y no eterna ${ }^{17}$.

La propuesta de Clarke crea un interesante cambio de dirección. Si la inmensidad, espacio o extensión, se deriva de la existencia necesaria, no será más un concepto propio de lo material contingente, sino que será, como Clarke le dice a Leibniz en su Cuarta Respuesta, más sustante que las sustancias contingentes aunque no es ni sustancia ni accidente; no obstante, al ser atributo de la divinidad, tiene mayor necesidad que lo contingente, aunque no es un ente alterno a Dios ni comparte su absoluta necesidad ${ }^{18}$.

${ }^{16}$ Clarke, Samuel, $A$ Dircourse Concerning the being and Attributes of God, Prop. III, p. 528.

${ }^{17}$ Clarke, Samuel, op. cit., Prop. III, pp. 528-9. "Este argumento de los cartesianos, que suponen que la idea de inmensidad es la idea de materia, los ha tenido muy perplejos (porque no sólo en las palabras se han contradicho a sí mismos, sino también en la realidad) han sido llevados fácilmente al intolerable absurdo de aseverar que la materia es un ser necesario".

${ }^{18}$ Leibniz-Clarke Correspondence. (C-L IV, 10, p.47). 
Surge así, de la existencia necesaria de Dios, una entidad (extensión o espacio) que no está ni en el reino de lo contingente material (como todo lo creado) ni en el de lo necesario divino, pero que sirvió de base a ciertos desarrollos de la ciencia físico-matemática.

Para Clarke, la infinitud es un concepto primario que está en nuestra mente y que no podemos desechar, aun haciendo abstracción de la idea de Dios. Así, no podemos dejar de imaginar inmensidad y eternidad como nociones abstractas ${ }^{19}$.

Ello significa que no llegamos al concepto de infinitud vía la experiencia o a través de las matemáticas per se; esto es, no obtenemos tal concepto a partir de lo cuantificable porque Clarke nos dice, expresamente, que ningún número o cantidad puede ser parte del infinito, puesto que no hay proporción entre las partes finitas y lo infinito ${ }^{20}$. Así, no podemos decir que espacio y tiempo son infinitos, en el sentido de la suma de sus partes finitas; más bien, lo que vemos es la propuesta de la infinitud y la eternidad cualitativas $^{21}$ en tanto se postulan, como veremos, como simples y absolutas.

Cuando hablamos del cambio de giro que imprime Clarke al problema, nos referimos sobre todo al tratamiento del espacio y el tiempo como simples. Así, en la proposición IV, Clarke nos dice que:

El espacio infinito es la inmensidad abstracta o la infinitud abstracta así como la infinita duración [tiempo infinito] es la eternidad abstracta ${ }^{22}$.

En el sentido ontológico, el espacio se despega completamente de la materia pues ésta es finita, tiene partes, es corruptible, divisible, se mueve, en una palabra, es compuesta, en tanto que el espacio es inmóvil, sin partes, indivisible, incorruptible, en una palabra, simple. De este modo, en el universo coexisten el espacio y el tiempo simples, más necesarios que las sustancias contingentes, porque ontológicamente son anteriores a lo

${ }^{19}$ Cfr. Clarke, Samuel, op. cit., Prop. III, p. 528.

${ }^{20} \mathrm{Ibidem}, \mathrm{p} .536$.

${ }^{21}$ Esta caracterización me la ha sugerido Carmen Silva, quien divide a los autores que estudian las nociones de espacio y tiempo, en quienes lo hacen cualitativamente y quienes lo hacen cuantitativamente.

22 Ibid., Prop. IV, p. 538. 
estrictamente creado, de materia compuesta y divisible. Así, espacio y tiempo eternos son el telón común de fondo a todo lo existente. Naturalmente, los seres finitos sólo pueden estar presentes en un tiempo y en un lugar a la vez, en tanto que:

La causa suprema, por ser esencia infinita máximamente simple, está en todo tiempo igualmente presente, tanto en su esencia simple, como en el ejercicio de todos sus atributos en todo punto de la inmensidad ilimitada, como si fuera realmente todo, sólo un punto ${ }^{23}$.

Dios está presente al universo y actúa sobre él a través de los simples espacio y tiempo. Las ideas de espacio y tiempo infinitos y simples, aunque con sus diferencias, son cercanas a los conceptos de espacio y tiempo absolutos en Newton.

Cuando Newton leyó el texto editado por Clarke, de su polémica con Leibniz, según lo refiere Koyré, le pidió a Des Maizeaux que, en su edición al francés, introdujera una 'advertencia', como propia de Clarke, que resulta ilustrativa a este respecto:

M. Clarke ha deseado que yo advierta a sus lectores que, en tanto que habla de espacio infinito o de inmensidad o de duración infinita o de eternidad y les da, por una inevitable imperfección del lenguaje, el nombre de cualidades o propiedades de la sustancia que es inmensa o eterna, no pretende entender el término de cualidad o propiedad en el mismo sentido que lo toman ordinariamente los que tratan de la lógica o la metafísica, en tanto que ellos lo aplican a la materia; sino que, con ello, él sólo quiere decir que el espacio y la duración son modos de la existencia en todos los seres, y modos infinitos y las consecuencias de la existencia de la sustancia que es realmente necesaria y sustancialmente omnipresente y eterna ${ }^{24}$.

El matiz que esta advertencia introduce es muy importante, porque no se trata ya de las atribuciones a una sustancia. Newton hace decir a Des Maizeaux que Dios no es una sustancia más, sino que su existencia es la sustancia con todos sus atributos, cualidades y propiedades; donde espacio y duración son modos de esa existencia, esto es, maneras de ser del existente

${ }^{23}$ Ibid., Prop. VI, p. 541.

${ }^{24}$ Koyré y Cohen, op. cit., p. 71 y pp. 83-84. 
de suyo, tan íntimamente ligadas, que no podemos rechazarlas sin rechazar la existencia de Dios $^{25}$.

Aunque efectivamente hay una diferencia entre propiedad y modo puesto que éste último queda más directamente relacionado con la existencia del ser supremo, con todo, subsiste el dilema para Clarke-Newton, según sus adversarios, o de 'la divinización del espacio' o de la 'espacialización de Dios'26.

Sin embargo, las acusaciones de Leibniz y Berkeley no parecen del todo fundadas, pues Clarke tuvo cuidado de establecer que el espacio infinito no es Dios, puesto que se trata de una entidad relativa a la existencia de Dios, pero que no se identifica con Él, ni tampoco es realmente independiente de Él. El problema surge, precisamente, de haber dotado al espacio y al tiempo de simplicidad, lo cual no significa que sean realmente sustancias ni tampoco estrictamente atribuciones, algo que Clarke toma de Newton para responder a Leibniz:

El espacio no es un ser, un ser eterno e infinito, sino una propiedad o consecuencia de la existencia de un ser eterno e infinito ${ }^{27}$.

Como ya lo señalé, las nociones abstractas de inmensidad infinita (espacio) y eternidad infinita (tiempo) se relacionan íntimamente con las nociones de espacio y tiempo absolutos de Newton. Con todo, Clarke no puede dejar a un lado la terminología de propiedad y cualidad. Pero, a pesar de la confusión terminológica, lo importante es que intenta precisar la entidad de espacio y de tiempo cuando afirma:

El espacio es inmenso e inmutable y eterno, igual que la duración. Así, nada es eterno fuera de Dios pues espacio y duración no están fuera de

\section{${ }^{25}$ Ibid., p. 84.}

${ }^{26}$ Ibidem. Aquí Koyré nos dice que Berkeley, en 1710 en los Principles of Human Knowledge, señala que a los newtonianos su posición los lleva a un dilema: o bien aceptar que el espacio real es Dios o bien que hay algo con Dios [aquí Berkeley se refiere a Raphson] que es eterno e increado, infinito, indivisible e inmutable, ambas alternativas perniciosas y nociones absurdas.

27 Ibid., p. 89. Newton lo destaca cuando dice que: "El espacio tiene su propio modo de existencia, que no compete ni a la sustancia ni al accidente". Cfr. Ibid., p. 88. 
Dios, sino que son causados por y son consecuencias inmediatas $\mathrm{y}$ necesarias de su existencia ${ }^{28}$.

Así, en este intento de clarificación, los newtonianos buscan, a la par que mantener el carácter de simplicidad de tiempo y espacio, en vista de su relación directa con la existencia divina, mostrar que no se colapsan con la divinidad ni son completamente independientes de ella. En mi opinión, se trata de una ardua lucha por otorgar a tiempo y espacio un estatuto ontológico muy peculiar, en el que desembocaron los "vacuismos" de siglos anteriores y que, merced a este extraño estatuto, logró una expresión matemática demostrable y aplicable.

A este respecto, una observación que me parece pertinente es que las nociones de tiempo y espacio, así configuradas, no sólo permitieron el desarrollo de una nueva física, sino que, habiendo sido instauradas como simples con tanta dificultad frente a la resistencia de los esquemas tradicionales, se erigen, luego, como paradigmas de simplicidad en el propio pensamiento de sus autores.

\section{El alma como sustancia extensa indivisible}

Que espacio y tiempo se toman como paradigmas de simplicidad, se hace obvio cuando Clarke, para mostrar la simplicidad del alma, nos remite a la noción simple de espacio como una prueba contundente y científica de que tales entidades (espirituales-extensas) son pensables. Así, en su Respuesta a Leibniz, quien le preguntaba ¿cómo una sustancia inmaterial, como el alma, puede ser indivisible si se asume que es extensa?, dice que, si puede probarse demostrativamente que las partes del espacio son 'absolutamente' indiscernibles', no puede ser mayor dificultad imaginarse que:

Todas las sustancias inmateriales pensantes (sobre la suposición de que la expansión no está excluida de su idea) deben ser muy semejantes ${ }^{29}$.

Para Vailati, el espacio clarkeano es extenso pero indivisible, porque en esta extensión las partes no lo son propiamente, sino que dependen unas

\footnotetext{
${ }^{28}$ Ibid., p. 90.

${ }^{29}$ Vailat, Ezio, op. cit., p. 394.
} 
de las otras para su existencia, en vista de su infinitud y de que no es posible, sin contradicción, pensar en su separación ${ }^{30}$.

Para nuestro estudio, lo importante de esta concepción es que, según Clarke, el alma tiene un estatuto análogo, esto es, hay que concebirla como una sustancia cuyas partes dependen unas de las otras al modo del espacio, i.e., como unitaria y simple, en vista de que la conciencia sólo puede inherir en un sujeto individual y no en un compuesto: de ahí que inhiera en el espíritu y no en la materia ${ }^{31}$.

Vailati señala a este respecto un problema interesante. Clarke parece mantener que la simplicidad del alma surge, como la del espacio, de su indivisibilidad (si las partes son realmente semejantes, no se pueden separar); pero si la extensión consiste en tener partes, sean o no separables, el alma tendrá partes $\mathrm{y}$, por consiguiente, no será un sujeto adecuado de inherencia de la conciencia. En suma, la indivisibilidad no garantiza la unidad de la conciencia, algo que Leibniz le señaló atinadamente a Clarke ${ }^{32}$.

Con relación a este problema hay que hacer, al menos, dos consideraciones. La primera es que, al usar el símil del espacio, Clarke tiene dificultades, porque al paso que de algo no activo como el espacio y sus partes semejantes, puede predicarse la homogeneidad y el isotropismo, cuando se habla del alma nos enfrentamos a una entidad sustantiva que realiza operaciones. La segunda consideración es que resulta sorprendente que Clarke dejase que se le colara una noción de extensión, tan semejante a la de los cuerpos que, aunque "mal llamadas", se caracteriza por tener partes.

Posiblemente la razón para mantener la "indiscernibilidad" de las "mal llamadas" partes o, en este sentido, la uniformidad para satisfacer el requisito de simplicidad, tiene que ver con la acción del alma en un tiempo y en un lugar. La localización concreta de la acción determina que el alma, aunque uniforme, sea extensa, de igual modo que el espacio uniforme, a través del cual Dios ejerce su actividad en el universo, sea igualmente extenso, esto es, con partes aunque isotrópico.

${ }^{30}$ Este argumento, según me señala José A. Robles, ya había sido usado por Patrizi.

${ }^{31}$ Cfr. Vailati, op. cit., p. 395.

${ }^{32}$ Cfr. Leibniz-Clarke Correspondence. (C-L V, 1-8). 
En realidad, la extensión se decanta en lo que hace a seres no corpóreos. No se trata de burdas partes diferenciadas, sino de partes semejantes e inseparables que permiten, tanto la manifestación consciente del hombre (en el caso del alma), como la actividad divina en el universo (en el caso del espacio y del tiempo). Por ello, en su Quinta Respuesta a Leibniz, Clarke dice:

Que, el que alma como el espacio sea extensa, implicaría que tiene partes, pero no que tiene partes separables, pues el alma es esencialmente un individuo y cuando ve, oye o piensa, lo hace como un todo ${ }^{33}$.

El alma es una totalidad en la que el todo antecede, lógicamente, a sus partes ya que algunas de sus potencias, en tanto todo, no pueden depender de sus partes, particularmente la conciencia que se fragmentaría, como apunta Vailati. Así, el alma exige la simplicidad. no sólo para dar cuenta del funcionamiento unitario de la conciencia, sino para poder aseverar su inmortalidad.

En suma, al admitir la extensión del alma e implícitamente partes en ella aun cuando sean indiscernibles e inseparables, Clarke hace un peculiar uso del argumento de simplicidad, merced a su concepción de la acción, que le orilla a afirmar, cercano a la paradoja, que el alma es a la par simple y extensa.

\section{Conclusión}

Considero que la fecundidad del argumento de simplicidad ha quedado delineada en función del problema de la infinitud de Dios y de sus modos de existencia eternidad e inmensidad. Hablar de Dios extenso, pero sin partes, en razón de su naturaleza espiritual, fue metafísicamente equivalente a postular un espacio absoluto cuyas partes son indiscernibles $\mathrm{e}$ inseparables. Por otra parte, la necesidad de proponer, desde la perspectiva de la física, espacio y tiempo como simples, acercaron a los newtonianos a un peligroso deísmo al caracterizar, en tanto simples, de manera muy semejante a Dios y al espacio-tiempo. En efecto, como simples, comparten la indivisibilidad, la incorruptibilidad, la inmovilidad, etc. aunque, por supuesto, espacio y tiempo no son sustancias per se, puesto que sólo son

${ }^{33}$ Ibidem (C-L V, 1-8). 
modos de existir del ser necesario y su estatuto ontológico es muy peculiar, pues tampoco son accidentes $y$, sin embargo, se sitúan más cerca de la necesidad que de la contingencia. La cercanía entre Dios y la inmensidad del espacio donde se ubica su creación, dio pie a que el ala Tory del parlamento considerara al newtonianismo una secta y, a los divines (teólogos) cercanos a Newton, como heterodoxos o herejes. Así, la encrucijada en que nació la ciencia nueva tiene que ver con una revolución intelectual, en lucha contra un pensamiento conservador y contra "la inevitable imperfección del lenguaje".

\section{Bibliografía}

1. Clarke, Samuel, "A Discourse Concerning the Being and Attributes of God" en Samuel Clarke The Works 1738, Garland Publishing Inc., Nueva York-Londres, 1978.

2. Gay, John H., "Matter and Freedom in the Thought of Samuel Clarke", en Journal of the History of Ideas, Vol. 24, Temple University, Philadelphia,

3. Koyré, Alexandre y Cohen, Bernard, "Newton et la correspondance Leibniz-Clarke" en Archive International d Histoire des Sciences, 15, 1962.

4. The Leibniz-Clarke Correspondence, Ed. H. G. Alexander, Manchester University Press, Barnes \& Noble, Nueva York, 1956.

5. Mijuskovicz, Ben Lazare, The Achilles of Rationalists Arguments, Martinus Nijhoff, La Haya, 1974.

6. Rodney, M. Joel, “Clarke, Samuel”, en Dictionary of Scientific Biograpby, Vol. 3, Charles Scribner's Sons, Nueva York, 1981.

7. Vailati, Ezio, "Clarke's Extended Soul", en Journal of the History of Philosopby. 31, julio 1993.

\section{Resumen}

La autora de este estudio se propone poner de relieve la importancia del argumento de la simplicidad en el desarrollo de las ideas de espacio y tiempo en Samuel Clarke y su incidencia en el newtonianismo. Así, en primer lugar intenta poner al descubierto la importancia que la noción de simplicidad tiene para el dualismo de Samuel Clarke, en donde se ponen en conexión la extensión y la simplicidad cuando se considera que la inmensidad de Dios es extensión indivisa. Por otra parte, recorre las vicisitudes del argumento de la simplicidad en función de las nociones físicas de espacio y de tiempo, para concluir que los simples de Clarke (espacio y tiempo) son muy cercanos a los conceptos de espacio y tiempo absolutos en Newton. Finalmente se explica cómo en opinión de Clarke el alma humana, siendo una 
sustancia simple y, por tanto, indivisible, es sin embargo una sustancia extensa; en este caso las partes, como las concibe Clarke, no son burdas y diferenciadas, sino semejantes e inseparables, algo que Leibniz habría reducido a una única entidad, en vista de su argumento de la identidad de los indiscernibles.

Palabras clave: "infinitud", "extensión espiritual", "indicernibilidad", "simplicidad", "vacuismo/plenismo"

\begin{abstract}
The author of this study intends to point out the importance of the argument of simplicity in the development of the ideas of space and time in Samuel Clarke and their incidence in newtonianism. Thus, she first intends to uncover the importance that the notion of simplicity possesses for Samuel Clark's dualism, in which extension and simplicity connect with one another, when we consider that God's immensity is indivisible extension. On the other hand, she goes through the vicissitudes of the argument of simplicity with regard to the physical notions of space and time, to conclude that Clarke's simples (space and time) stand very near the concepts of absolute space and time in Newton. Finally, an explanation comes of how, in Clarke's opinion, the human soul, being a simple substance, and therefore indivisible, is nevertheless an extensive substance; in this case, the parts, as Clarke conceives them, are not coarse and differentiated, but alike and inseparable, which Leibnitz is thought to have reduced to a single entity, in view of his argument of the identity of indiscernibles.
\end{abstract}

Key words: "infiniteness", "spiritual extension", "indiscernibility", "simplicity", "vacuity/plenitude". 